\title{
The Effect of Femoral Nerve Block Versus Adductor Canal Block on the Quality of early Recovery after Revision Total Knee Arthroplasty, a retrospective Study
}

Aboud AlJa'bari ( $\nabla_{\text {j_aboud@hotmail.com ) }}$

DMF hospital

Khalid AL Sheikh

DMF hospital

\section{Research Article}

Keywords:

Posted Date: March 3rd, 2022

DOI: https://doi.org/10.21203/rs.3.rs-1079221/v3

License: (a) This work is licensed under a Creative Commons Attribution 4.0 International License. Read Full License 


\section{Abstract}

Background: Multimodal pain analgesia strategies are common in perioperative management of total knee arthroplasty (TKA), although the role of adductor canal blocks (ACB) versus femoral nerve block on early postoperative recovery for revision knee surgery is not investigated.

Aim: The purpose of this study is to independently evaluate the effect of ACB on short-term postoperative outcomes including (1) length of stay (LOS), (2) postoperative narcotic utilization, and (3) function with physical therapy in revision TKA.

Methods: We retrospectively identified a cohort study of consecutive 40 patients from January 2021 to July 2021 who had undergone unilateral revision TKA using a single-shot ACB (19 patients) vs femoral nerve block (21 patients) under spinal anesthesia (hyperbaric 0.5\% Marcaine $2.5 \mathrm{ml}$ and 20 microgram fentanyl) in addition to a standardized multimodal pain analgesia protocol. These 2 groups were compared using independent sample t-tests with primary end points of interest being distance ambulated after surgery, and inpatient narcotic use.

Results: Quadriceps strength was better preserved in adductor group than in femoral group. Walking meters and going upstairs were better results in adductor group. IV morphine consumption within the first 48 hours period were less in adductor group comparing to femoral group.

Conclusion: Adductor nerve block showed better early recovery in revision TKA when comparing to femoral nerve block (FNB).

\section{Primary Outcome Measure}

Our primary objective is to determine if ACBs will result in improved quality of recovery compared with FNBs (within 24 hours).

\section{Secondary Outcome Measures:}

1. Opioid consumption Time Frame: 48 hours post-operatively.

Total opioid consumption during this period after surgery will be morphine equivalent doses and calculated on a milligram until discharge.

2. Pain score measurement: we will use Numeric rating scale for measurement (NRS) Time Frame: 48 hours post-op

Pain scores on a $0-10$ numerical rating scale (NRS) where $0=$ no pain and $10=$ the worst pain ever. Pain scores will be assessed every 8 hours from nursing documentation, through 48 hours or until discharge, whichever occurs first.

\section{Criteria:}


Inclusion Criteria

Adult male or female patients ages 18-80 scheduled for elective revision TKA

American Society of Anesthesiologists (ASA) physical status I -III

Mentally competent and able to give consent for enrollment in the study

Exclusion Criteria:

Patients with any contraindications to regional anesthesia such as infection, nerve injury, anticoagulation

Patient refusal

Patients with an allergy to local anesthetics or systemic opioids

\section{Introduction}

In recent years, the point of care in acute pain analgesia strategies following total knee arthroplasty has dramatically shifted from femoral nerve block to adductor canal block [1, 2].

There are several studies supporting adductor canal nerve block as an alternative for postoperative analgesia after total knee arthroplasty, but as far as we know, this is the first study to investigate unilateral revision TKA. This novel block has demonstrated equivalent analgesic efficacy compared to femoral nerve block, with the benefit of reducing quadriceps weakness significantly less than femoral nerve block, thus enhancing earlier active mobilization $[2,3]$.

Focusing on enhance recover after surgery, adductor canal block could be considered as preventing factor from complications such as deep vein thrombosis and joint rigidity resulting from the lack of early mobilization. These advances may potentially result in decreasing the total length of hospital stay and therefore a reduction in associated health care costs $[4,5]$.

Conventional systemic opioid therapy has been the primary modality to control pain after TKA. However, post-operative analgesia has clinically improved over the past decade due to perioperative multimodal medications [6-8], local infiltration analgesia [7, 9], and the advancement of regional anesthetic techniques.

The focus of peripheral nerve blocks has changed our practice in the setting of acute pain management following TKA from continuous lumbar epidural analgesia [10], to single injection or continuous Femoral Nerve Block (FNB) [10], and more recently, to the Adductor Canal Block.[9]

The main purpose of this study is to independently evaluate the effect of ACB on short-term postoperative outcomes including (1) early enhanced recovery with physiotherapy, (2) postoperative narcotic utilization, and (3) secondary side effects such as PONV. 


\section{Methods}

The study is approved by DMF hospital ethical committee and was performed in accordance with Helsinki declaration, our institutional database was used to retrospectively identify 2 cohort groups of consecutive patients who had undergone unilateral revision TKA.

Patients who underwent surgery from January 2021 through July 2021 were included, as we follow our hospital specific policy for revision TKA perioperative protocol, including use of tranexamic acid $(1 \mathrm{~g} 30$ minutes before incision), peri-capsular injection ( $0.2 \%$ ropivacaine at the end of procedure injected by surgeon), and inpatient postoperative physiotherapy. Patients undergoing single-sided revision TKA under spinal anesthesia were included. Exclusion criteria included simultaneous bilateral TKA as single surgery, second-stage treatment for infection, or incomplete data recorded postoperatively.

All 40 patients in the retrospective study received a multimodal pain protocol including oral morphine, anti-emetics, paracetamol and anti-inflammatory unless contraindicated.

All patients received, in the perioperative stage, single shot of ACB or femoral consisting of $0.5 \%$ ropivacaine $20 \mathrm{ml}$ without epinephrine performed by our regional anesthetists. Blocks were performed under ultrasound guidance. Anesthetic doses were based on patient body mass index (BMI) and age. These cohorts did not statistically differ in terms of gender, BMI, or Age and duration of surgery or anesthesia. (Table 1)

Table 1

Demographic data

\begin{tabular}{|llll|}
\hline & Adductor group & Femoral group & P value \\
\hline Age & $66(62-70)$ & $70(68-76)$ & 0.98 \\
\hline Gender (female) & $9 / 19$ & $13 / 21$ & 0.36 \\
\hline ASA & $2(2.1-2.6)$ & $2(2.5-2.7)$ & 0.50 \\
\hline Duration of surgery (min) & $142(135-167)$ & $155(125-165)$ & 0.34 \\
\hline BMl & $29(27-31)$ & $30(27-32)$ & 0.69 \\
\hline Side of TKA (right) & $12 / 19$ & $14 / 21$ & 0.82 \\
\hline
\end{tabular}

Our orthopedic surgeon is transitioned his practice to cemented components without tourniquet to all patients included in this study.

Retrospective chart review collected perioperative data for both groups. Our points of interest were quadriceps strength and patient's ability to walking meters and upstairs with physical therapy on postoperative day 1 (POD1), and postoperative narcotic use in morphine equivalents per day (IV morphine mg per day). The criteria for discharge after revision TKA at our institution include pain adequately controlled on oral medications, tolerating oral intake, voiding, and being cleared by physiotherapy if the 
patient is able to perform antigravity leg lifts, bed mobility joints coach, and ability to ambulate 50 meters independently.

Nurses, therapists, and case managers had no knowledge of the use of ACB peri-operatively and made all decisions on discharge criteria and pain medication administration.

\section{Statistical analysis:}

Analysis was performed on the 2 groups using independent sample $t$-tests, and chi-square analyses were used for categorical data where appropriate. $P$ value of $<.05$ was set as the threshold to determine statistical significance of results. All statistical analyses were completed using JMP version 14 .

\section{Results}

The results of our study along with the inconclusive nature of the current literature reviewed above have led the senior authors to a more selective use of ACB after revision TKA. It is an expense that has failed to definitively demonstrate a clinically relevant difference in perioperative pain control or outcomes we are now selectively using in patients known to have preoperative issues with pain control or stiffness.

Our study was aimed at the immediate postoperative period and thus we were unable to collect data after patients discharge from the hospital. Furthermore, despite statistically significant differences in quadriceps strength 3 vs $2(P=.0045)$ and distance ambulated with physiotherapist 52 meters vs 33 meters $(P=.0063)$, and more patients in adductor group able to walk upstairs $11 / 19$ versus $6 / 21$ in femoral group with $P=0.0024$. (Table 2 )

Table 2

Postoperative outcomes on early rehabilitation

\begin{tabular}{|llll|}
\hline & Adductor & Femoral & P value \\
\hline Quadriceps strength & $3(2.5-3)$ & $2(2-2.8)$ & $0.045^{*}$ \\
\hline Walking in meters & $52(41-62)$ & $33(24-43)$ & $0.0063^{*}$ \\
\hline Walking upstairs (yes) & $11 / 19$ & $6 / 21$ & $0.0024^{*}$ \\
\hline *P value < $0.5 \%$ is significant & & \\
\hline
\end{tabular}

Our study showed lower IV morphine consumption 24hours postoperatively in adductor group $8.8 \mathrm{mg}$ vs $14.4 \mathrm{mg}$ in femoral group $(P=0.04)$ and 48 hours postoperatively 9 vs $17 \mathrm{mg}(P=0.043)$ respectively. (Table 3) 
Table 3

Secondary outcomes

\begin{tabular}{|llll|}
\hline & Adductor group & Femoral group & P value \\
\hline 24 hours IV morphine consumption $(\mathrm{mg})$ & $8.8(4.8-12.7)$ & $14.4(10.7-18)$ & $0.04^{\star}$ \\
\hline 48 hours IV morphine consumption (mg) & $9(1.6-12.3)$ & $17(8-26)$ & $0.043^{*}$ \\
\hline Post-operative nausea \& vomiting & $1 / 19$ & $4 / 21$ & $0.0002^{*}$ \\
\hline Rest Pain score 24 hours & $0(0-1)$ & $1.5(1.3-1.8)$ & $0.012^{\star}$ \\
\hline Pain score on movement & $3.4(2.5-4.2)$ & $3.8(3-4.5)$ & 0.466 \\
\hline *P value < 0.5\% is significant & & & \\
\hline
\end{tabular}

\section{Discussion}

The positive evolution of regional anesthesia on surgical outcome has important impact on enhanced recovery. In recent years, the focus of acute pain analgesia strategies following total knee arthroplasty has changed our practice from femoral nerve block to adductor canal block.

There are several studies supporting the adductor canal nerve block as an alternative for postoperative analgesia after total knee arthroplasty. Based on our study results, we recommend that an adductor canal block could replace a femoral nerve block as the primary regional analgesic following total knee arthroplasty.

Pain following TKA can result in diminished function with physical therapy, decreased mobilization, prolonged hospitalization, and reduced patient satisfaction. FNB and ACB have both been implicated as important components in multimodal analgesia protocol.

To our knowledge, this is the first retrospective study exploring the role of ACB versus FNB in revision TKA. We demonstrated improved function with physical therapy and decreased length of hospitalization when ACB compared to FNB; however, we showed significant difference in postoperative in hospital intravenous narcotic use between the 2 groups on the 48 hours period postoperatively. (Table 3 )

Femoral nerve blocks (FNB) have been shown in many studies to reduce opioid consumption and decrease postoperative pain scores [9]. Unfortunately, FNB is associated with motor blockade of the quadriceps muscle which impair postoperative mobilization and increase risks of falls [10]. Adductor canal blocks (ACB) provide a more distal nerve blockade, at the mid-thigh, mainly providing sensory blockade in the distribution of the saphenous nerve while sparing quadriceps function. Literature has supported improved motor function with similar pain control when comparing ACB to FNB [6], [7], [8], [9].

The results of our retrospective study were like those found in a recent meta-analysis by Ma et al [11], where combined ACB with PAI was associated with improved postoperative mobilization on POD1 but 
that did not decrease the narcotic consumption or hospital length of stay.

This study encouraged us to perform controlled randomized triple blinded study for larger number of patients. Our study is not without limitations. First, these results are from a single center, small sample. Finally, given the retrospective nature of the study, we did not have a "control group" cohort to include as all patients received regional anesthesia.

Additionally, our study is retrospective in nature and we were able to collect consistent verbal analog scale pain score in the postoperative period were consistently recorded at varying time intervals.

\section{Abbreviations}

FNB -femoral nerve block

ACB -adductor canal block

TKA - total knee arthroplasty

PONV-postoperative nausea and vomiting

\section{Declarations}

Ethics approval: The study is approved by DMF hospital ethical committee and was performed in accordance with Helsinki declaration.

Consent to participate: Informed consent was obtained from all participants.

Consent for publication: not applicable.

Availability of data and material: The database generated and analyzed during the current study are not publicly available due to our hospital policy of patient's confidentiality is preserved but are available from the corresponding author on reasonable request.

Competing interests: no competing interests.

Funding: no funding.

Authors' contributions: A. AJ has collected the data, wrote the manuscript, did the statistical analysis, and follow up the patients. K.S has helped in writing the manuscript.

Acknowledgments: no acknowledgments.

\section{References}


1. -P. Cram, X. Lu, S.L. Kates, J.A. Singh, Y. Li, B.R. Wolf Total knee arthroplasty volume utilization, and outcomes among Medicare beneficiaries, 1991-2010 JAMA, 308 (2012), pp. 1227-1236

2. -S. Kurtz, K. Ong, E. Lau, F. Mowat, M. Halpern Projections of primary and revision hip and knee arthroplasty in the United States from 2005 to 2030 J Bone Joint Surg Am, 89 (2007), pp. 780-785

3. -H. Husted, T.H. Lunn, A. Troelsen, L. Gaarn-Larsen, B.B. Kristensen, H. Kehlet. Why still in hospital after fast-track hip and knee arthroplasty? Acta Orthop, 82 (2011), pp. 679-684

4. Ibrahim, M.S., Khan, M.A., Nizam, I., et al. Peri-operative interventions producing better functional outcomes and enhanced recovery following total hip and knee arthroplasty: an evidence-based review. (2013) BMC Med 11: 37.

5. Kehlet, H., Thienpont, E. Fast-track knee arthroplasty - status and future challenges. (2013) Knee 20 (S1, S29): 33.

6. .Tank, P.W. Grant's Dissector. (2005)13 edition Lippincott Williams \& Wilkins .6

7. Tubbs, R.S., Loukas, M., Shoja, M.M., et al. Anatomy and potential clinical significance of the vast adductor membrane. (2007) Surg Radiol Anat 29(7): 569-57

8. .8. Andersen, H.L., Andersen, S.L., Tranum-Jensen, J. The spread of injectate during saphenous nerve block at the adductor canal: a cadaver study. (2015) Acta Anaesthesiol Scand 59(2): 238-245

9. Cowlishaw, P., Kotze, P. Adductor canal block-or subsartorial canal block? (2015) Reg

10. Anesth Pain Med 40(2): 175 - 17.10. Gardner, E. The innervation of the knee joint. (1948) Anat Rec 101(1): 109-130.

11. Ma J, Gao F, Sun W, Guo W, Li Z, Wang W. Combined adductor canal block with periarticular infiltration versus periarticular infiltration for analgesia after total knee arthroplasty. Medicine (Baltimore). 2016 Dec;95(52): e5701. 